\title{
Search for the Higgs boson in fermionic channels using the CMS detector
}

\author{
Jesús Vizán (on behalf of the CMS Collaboration)* \\ Centre for Cosmology, Particle Physics and Phenomenology (CP3) \\ Université catholique de Louvain \\ Chemin du Cyclotron, 2 \\ B-1348, Louvain-la-Neuve \\ Belgium \\ E-mail: Jesus.Manuel.Vizan.Garcia@cern.ch
}

An overview of the latest CMS results from searches for a Standard Model Higgs boson decaying to fermions is presented. The dataset used corresponds to an integrated luminosity of approximately $5 \mathrm{fb}^{-1}$ at a center-of-mass energy of $7 \mathrm{TeV}$ and $20 \mathrm{fb}^{-1}$ at $8 \mathrm{TeV}$. The main accessible Higgs boson fermionic decay modes with the current dataset at LHC are $H \rightarrow \tau \tau$ and $H \rightarrow b \bar{b}$. In the case of the Standard Model Higgs boson decaying to a tau pair an excess of data over the background-only hypothesis with a significance of 3.2 standard deviations is observed, while an excess with a significance of 2.1 standard deviations is observed for $b \bar{b}$ decays. The combination of both searches results in strong evidence for the direct coupling of the $125 \mathrm{GeV}$ Higgs boson to down-type fermions, with an observed significance of 3.8 standard deviations. Searches for these decay modes when the Higgs boson is produced in association with top quarks are presented separately. Searches for the Higgs boson decaying to a pair of muons or electrons are also presented.

XXII. International Workshop on Deep-Inelastic Scattering and Related Subjects, 28 April - 2 May 2014

Warsaw, Poland

\footnotetext{
* Speaker.
} 


\section{Introduction}

The discovery of the mechanism for electroweak symmetry breaking constitutes one of the main scientific goals of the LHC. In the standard model (SM), this symmetry breaking is achieved via the Brout-Englert-Higgs mechanism, leading to the prediction of a single observable particle referred to as the Higgs boson. In Summer of 2012 a new particle was observed by the CMS and ATLAS experiments with a mass of approximately $125 \mathrm{GeV}$ [1, 国, 的. To date, the measurements of the properties of this particle are compatible with the SM expectations in terms production rates, decay rates, and spin-parity quantum numbers [ [ , 5, 6], and significant signals have been reported in the bosonic decay channels $H \rightarrow \gamma \gamma, H \rightarrow W W$, and $H \rightarrow Z Z$. Establishing that the $125 \mathrm{GeV}$ boson decays also to fermions is essential for identifying this particle as the SM Higgs boson.

The predominant decay modes of the SM Higgs boson at $m_{H}=125 \mathrm{GeV}$ are $H \rightarrow \tau \tau$ and $H \rightarrow b \bar{b}$, and represent the best opportunity at LHC to observe directly the SM Higgs boson decay to down-type fermions. The searches for those decays have been performed and combined at CMS [7] using the full available 2011 and 2012 datasets, at 7 and $8 \mathrm{TeV}$, respectively. In addition, also a search for the more challenging $H \rightarrow \mu \mu$ channel, presenting a much smaller branching ratio, has been performed. The various searches make use of the main SM Higgs boson production modes at LHC; gluon-gluon fusion, vector-boson fusion (VBF), and associated production with a $\mathrm{W}$ or $\mathrm{Z}$ boson, or a $t \bar{t}$ pair. The $t \bar{t} H$ production mode is of particular interest as it represents the only opportunity to directly prove the $t \bar{t} H$ vertex at LHC without making assumptions on contributions beyond the SM. The fermionic $H \rightarrow \tau \tau$, and especially the $H \rightarrow b \bar{b}$ decays contribute to the sensitivity to this production mode, and are included in the combined search for $t \bar{t} H$ at CMS.

\section{Higgs boson produced in association with a top quark pair}

\section{1 $t \bar{t} H, H \rightarrow b \bar{b}$ and $H \rightarrow \tau \tau$}

The fermionic decays $H \rightarrow b \bar{b}$ and the $H \rightarrow \tau \tau$, when both taus decay hadronically are considered in the study of the $t \bar{t} H$ production [8]. The events are classified according to the decay of the associated top quarks as lepton+jet or dilepton events in the $H \rightarrow b \bar{b}$ case, while only lepton+jet events are considered for $H \rightarrow \tau \tau$. The top-quark pair production with additional jets is the main background of this search. Events are further categorized based on the number of reconstructed jets and b-tagged jets to increase the sensitivity of the analysis. Multivariate analysis techniques are used to discriminate between signal and background. No significant excess in the data is observed after a simultaneous fit of the multivariate output distribution in all the categories is performed, and 95\% confidence level (CL) upper limits are set on the possible presence of a SM signal. The observed (expected) limit for $m_{H}=125 \mathrm{GeV}$ at $\sqrt{s}=8 \mathrm{TeV}$ is 5.2 (4.1) times the SM prediction.

\section{$2.2 t \bar{t} H$ combination}

The mentioned fermionic ttH searches from the previous section are combined with other searches in this production mode with $H \rightarrow \gamma \gamma$ [9] and $H \rightarrow$ multileptons [10]. The combined observed upper limit on the signal strength parameter, $\mu=\sigma / \sigma_{S M}$, is 4.3 for a mass of $m_{H}=$ 

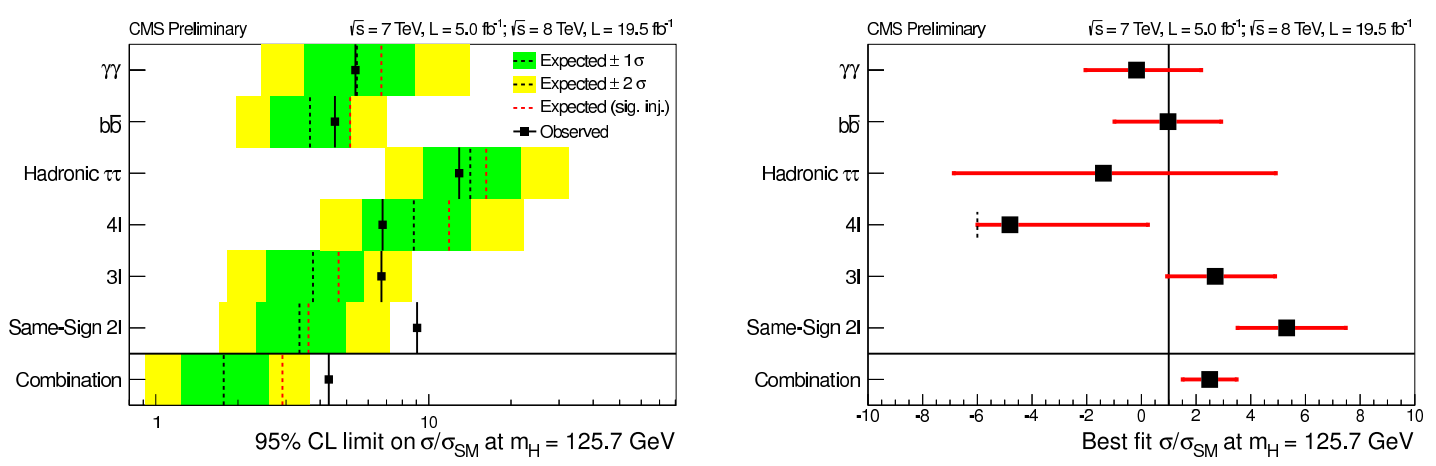

Figure 1: Left: The observed and expected 95\% CL upper limits on the signal strength parameter (left). Right: The best-fit values of the signal strength. The results are shown for the different channels and for the combined ttH search for $m_{H}=125.7 \mathrm{GeV}$.

125.7 GeV, while the expected limit is 1.8 . The signal strength is $2.5_{-1.1}^{+1.0}$. These results are shown in Figure 1 for the different considered channels and for the combination.

\section{Higgs boson decays to $b \bar{b}$}

\section{1 $V H, H \rightarrow b \bar{b}$}

The dominant decay mode of the Higgs boson at $m_{H}=125 \mathrm{GeV}$ is $H \rightarrow b \bar{b}$, with a branching fraction of $\approx 58 \%$ [11]. The irreducible background from QCD production of $b \bar{b}$ pairs is overwhelming for the main gluon-gluon fusion production mode. A search for $H \rightarrow b \bar{b}$ is performed however in events where the Higgs boson is produced in association with a $W$ or a $Z$ boson, as the presence of a vector boson in the final state results in a large reduction of the QCD background. This CMS search uses the available $5.1 \mathrm{fb}^{-1}$ of $7 \mathrm{TeV}$ data and $18.9 \mathrm{fb}^{-1}$ of $8 \mathrm{TeV}$ data [12]. The analysis is split in the following six categories based on the decay mode of the vector boson: $W(\mu v) H, W(e v) H, W(\tau v) H, Z(\mu \mu) H, Z(e e) H$, and $Z(v v) H$. The main backgrounds arise from production of vector bosons in association with jets, single top, $t \bar{t}$, diboson, and QCD multijet processes. A multivariate discriminator based on boosted decision trees is used to distinguish the signal from the different backgrounds. Upper limits on the $V H$ production cross section times the $H \rightarrow b \bar{b}$ branching fraction are extracted from a fit to the shape of the multivariate output. A broad excess of events is seen for the considered mass range $110-135 \mathrm{GeV}$. The excess has a local significance of 2.1 standard deviations at $m_{H}=125 \mathrm{GeV}$, with a best fit of the signal strength of $1.0 \pm 0.5$. These results are shown in Figure 2

\subsection{VBF $H \rightarrow b \bar{b}$}

A search for $H \rightarrow b \bar{b}$ with VBF production is performed using $\approx 19 \mathrm{fb}^{-1}$ of $8 \mathrm{TeV} \mathrm{CMS}$ data [13]. The signal extraction is derived from fits to the invariant mass distribution of the reconstructed $b \bar{b}$ pair. To improve the mass resolution a neural network regression technique is employed. The sensitivity of the analysis is improved by classifying the events based on a neural 

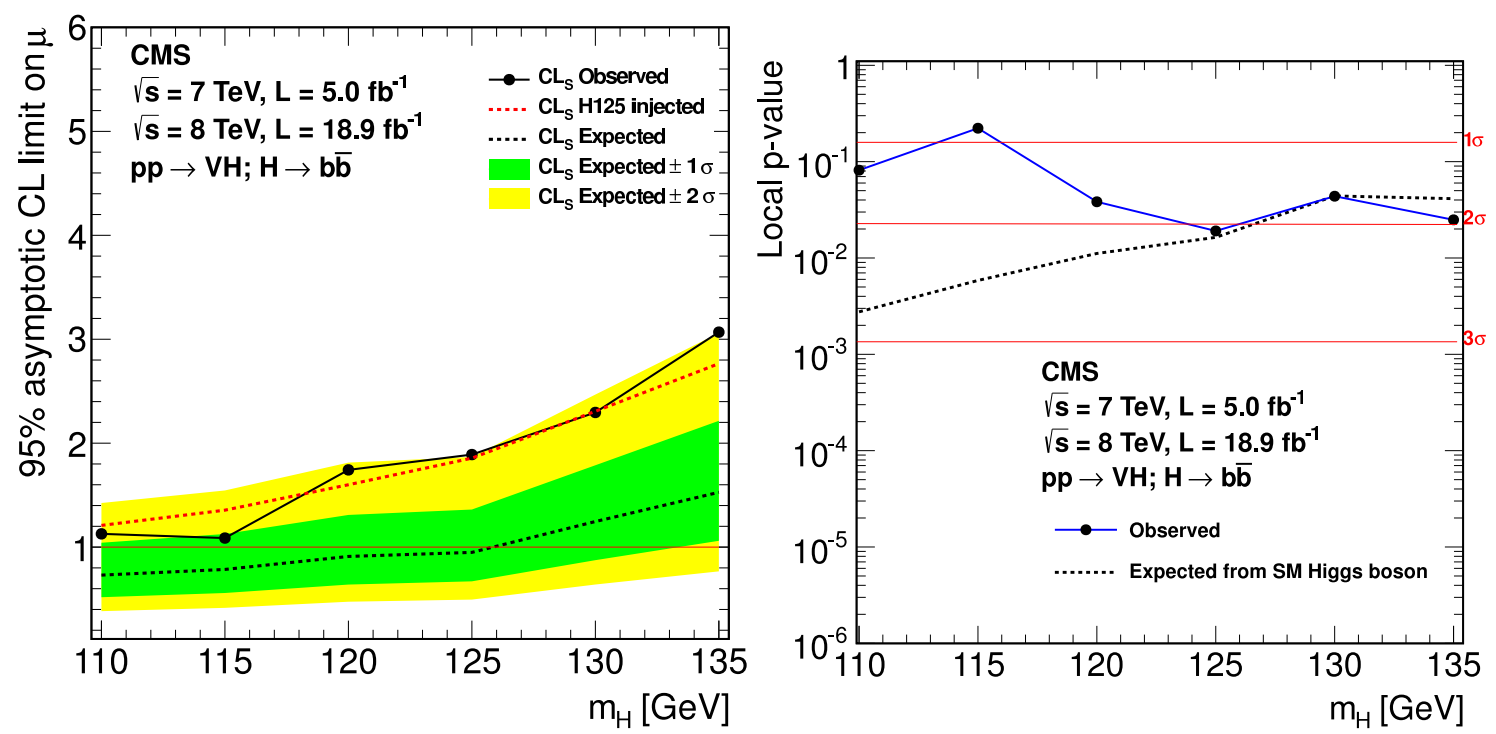

Figure 2: Left: The observed and expected 95\% CL upper limits on the signal strength parameter (left). Right: Local p-value and significance in number of standard deviations as a function of the SM Higgs boson mass hypothesis. The results are shown for the $V H, H \rightarrow b \bar{b}$ search.

network trained to separate signal events from background ones, without making use of kinematic information of the two b-tagged jets. At $m_{H}=125 \mathrm{GeV}$ the expected upper 95\% CL limit on the signal strength is 3.0 , while the observed limit is 3.6. The fitted signal strength is $0.7 \pm 1.4$.

\section{Higgs boson decays to $\tau \tau$}

A CMS search for $H \rightarrow \tau \tau$ is performed using the full available dataset of $4.9 \mathrm{fb}^{-1}$ at $\sqrt{s}=$ $7 \mathrm{TeV}$ and $19.7 \mathrm{fb}^{-1}$ at $\sqrt{s}=8 \mathrm{TeV}$ [14]. The main production modes for this search are gluongluon fusion and VBF. All the six $\tau$-pair final states, $\mu \tau_{h}, e \tau_{h}, \tau_{h} \tau_{h}, e \mu, \mu \mu$, and $e e$ are considered. Events are categorized based on the number of reconstructed jets. The one-jet category is mainly sensitive to the gluon-gluon fusion production mode, while the two-jet category enhances the VBF production mode. The $\mathrm{VH}$ production mode is also targeted by requiring one or two additional electrons or muons compatible with $W$ or $Z$ boson decays. Eight additional channels are considered in this case, based on the decay products of the $W$ or $Z$ boson, and of the $\tau$-pair.

The signal is extracted from the distribution of the invariant mass of the $\tau$-lepton pair for most of the channels. This variable is computed using a likelihood-based method which takes as inputs kinematic variables related to the visible decay products and the missing transverse energy. This allows to achieve a better separation between the $H \rightarrow \tau \tau$ signal and the $Z \rightarrow \tau \tau$ background. An excess of events over the background-only hypothesis is observed with a local significance of 3.2 standard deviations at $m_{H}=125 \mathrm{GeV}$, while the expected significance is 3.7 standard deviations. The best-fit value for the signal strength is $0.78 \pm 0.27$ times the SM prediction. These results, presented in Figure 3, constitute evidence for the coupling of the $125 \mathrm{GeV}$ Higgs boson and the $\tau$ lepton. 

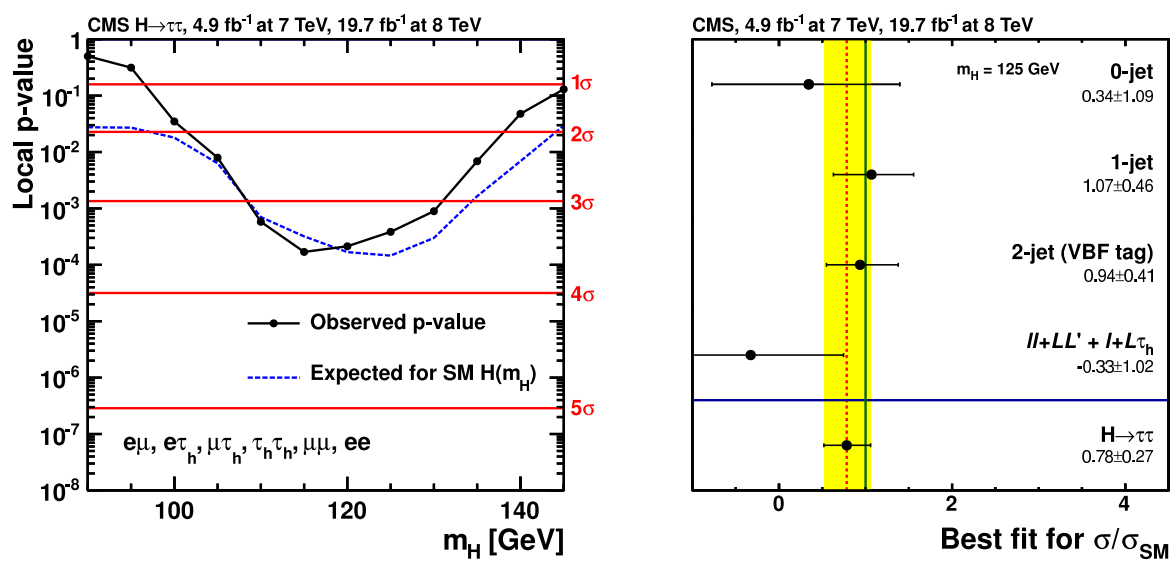

Figure 3: Left: Local p-value and significance in number of standard deviations as a function of the SM Higgs boson mass hypothesis. Right: The best-fit values of the signal strength for $m_{H}=125 \mathrm{GeV}$. The results are shown for the $H \rightarrow \tau \tau$ search.

\begin{tabular}{cccc}
\hline Channel & \multicolumn{2}{c}{ Significance $(\sigma)$} & Best-fit \\
$\left(m_{H}=125 \mathrm{GeV}\right)$ & Expected & Observed & $\mu$ \\
\hline$V H \rightarrow b \bar{b}$ & 2.3 & 2.1 & $1.0 \pm 0.5$ \\
$H \rightarrow \tau \tau$ & 3.7 & 3.2 & $0.78 \pm 0.27$ \\
\hline Combined & 4.4 & 3.8 & $0.83 \pm 0.24$
\end{tabular}

Table 1: Significance and best-fit value for the $H \rightarrow b \bar{b}$ and $H \rightarrow \tau \tau$ searches and their combination.

\section{5. $H \rightarrow b \bar{b}$ and $H \rightarrow \tau \tau$ combination}

The searches for $H \rightarrow b \bar{b}$ for vector-boson associated production, and $H \rightarrow \tau \tau$ presented in Sections 3.1 and $\emptyset$ are combined [15]. This combination results in strong evidence for the direct coupling of the $125 \mathrm{GeV}$ Higgs boson to down-type fermions, with an observed (expected) significance of 3.8 (4.4) standard deviations. The main results of the search are summarized in Table 1.

\section{Higgs boson decays to $\mu \mu$ and $e e$}

A CMS search for $H \rightarrow \mu \mu$ is performed with at total luminosity of $5.0 \mathrm{fb}^{-1}$ at $\sqrt{s}=7 \mathrm{TeV}$ and $19.7 \mathrm{fb}^{-1}$ at $\sqrt{s}=8 \mathrm{TeV}[16$. In the SM the decay $H \rightarrow \mu \mu$ presents a vary small branching ratio of $2.2 \times 10^{-4}$ at $m_{H}=125 \mathrm{GeV}$. However the search takes advantage of the clean signature in the detector and the excellent dimuon invariant mass resolution. Events are categorized based on the number or reconstructed jets attempting to separate the gluon-gluon fusion and VBF production components. The signal is extracted by means of a fit to the $m_{\mu \mu}$ distribution using signal and background shapes. Upper limits on the cross section times the $H \rightarrow \mu \mu$ branching ratio are set. The observed (expected) limit is found to be 7.4 (5.1) times the SM prediction.

A similar search for the SM Higgs boson in the rare decay mode ee is also performed. No excess of events over the background prediction is seen, and an upper limit of $0.038 \mathrm{pb}$ is set on the cross-section times branching ratio for $m_{H}=125 \mathrm{GeV}$ at $\sqrt{s}=8 \mathrm{TeV}$. 


\section{Summary}

Searches for a Standard Model Higgs boson decaying to fermions have been performed in CMS for different channels, including $b \bar{b}, \tau \tau$, and $\mu \mu$. Upper limits on the cross section times the branching ratio are set for the different decay modes at the $95 \%$ confidence level. Excesses in the $b \bar{b}$ and $\tau \tau$ modes are seen with significances of 2.1 and 3.2 standard deviations, respectively. The significance of the excess for the combined search is 3.8 standard deviations. This represents strong evidence for the direct coupling of the $125 \mathrm{GeV}$ Higgs boson to down-type fermions.

\section{References}

[1] CMS Collaboration, Observation of a new boson at a mass of $125 \mathrm{GeV}$ with the CMS experiment at the LHC, Phys. Lett. B 716 (2012) 30.

[2] CMS Collaboration, Observation of a new boson with mass near $125 \mathrm{GeV}$ in pp collisions at $\sqrt{s}=7$ and $8 \mathrm{TeV}, \mathrm{JHEP} 06$ (2013) 081.

[3] ATLAS Collaboration, Observation of a new particle in the search for the standard model Higgs boson with the ATLAS detector at the LHC, Phys. Lett. B 716 (2012) 1.

[4] ATLAS Collaboration, Evidence for the spin-O nature of the Higgs boson using ATLAS data, Phys. Lett. B 726 (2013) 120. arXiv:1307.1432.

[5] CMS Collaboration, Measurement of Higgs boson production and properties in the $W W$ decay channel with leptonic final states, JHEP 01 (2014) 096.

[6] CMS Collaboration, Measurement of the properties of a Higgs boson in the four-lepton final state, Phys. Rev. D 89 (2014) 092007.

[7] CMS Collaboration, The CMS experiment at the CERN LHC, JINST 3 (2008) S08004.

[8] CMS Collaboration, Search for Higgs Boson Production in Association with a Top-Quark Pair and Decaying to Bottom Quarks or Tau Leptons, CMS-PAS-HIG-13-019.

[9] CMS Collaboration, Search for $t t H$ production in events with $H \rightarrow \gamma \gamma$ at $\sqrt{s}=8 \mathrm{TeV}$ collisions, CMS-PAS-HIG-13-015.

[10] CMS Collaboration, Search for the standard model Higgs boson produced in association with top quarks in multilepton final states, CMS-PAS-HIG-13-020.

[11] S. Dittmaier et al. (LHC Higgs Cross Section Working Group), arXiv:1101.0593.

[12] CMS Collaboration, Search for the standard model Higgs boson produced in association with a W or a Z boson and decaying to bottom quarks, Phys. Rev. D 89 (2014) 012003.

[13] CMS Collaboration, Search for the standard model Higgs boson produced in vector boson fusion, and decaying to bottom quarks, CMS-PAS-HIG-13-011.

[14] CMS Collaboration, Evidence for the $125 \mathrm{GeV}$ Higgs boson decaying to a pair of $\tau$ leptons, JHEP 05 (2014) 104

[15] CMS Collaboration, Evidence for the direct decay of the $125 \mathrm{GeV}$ Higgs boson to fermions, Nature Physics (2014), doi:10.1038/nphys3005.

[16] CMS Collaboration, Search for the standard model Higgs boson in the dimuon decay channel in pp collisions at $\sqrt{s}=7$ and $8 \mathrm{TeV}$, CMS-PAS-HIG-13-007. 